\title{
Mental Health of Adolescents and Youth
}

\author{
Valentina Telhaj \\ European University of Tirana, Tirana, Albania
}

\begin{abstract}
The health and mental well-being of children and adolescents in general is good. Most are satisfied with their lives, perceive their health to be good, and do not regularly suffer from health complaints. The main problems of the first half of the 20th century, such as acute infections and high infant mortality, have diminished in importance. Instead of physical disorders, mental illness accounts for a large and growing share of ill health among children and adolescents in Europe. Including emotional problems, this may conduct problems and learning disabilities came to the fore in the middle of the last century. Currently, the frame of mental health and socio-economic influences on health has risen to achieve importance within child and adolescent health. World Health Organization (WHO) declared that young people's mental health is a key area of concern to which professionals and policy-makers must direct their attention. Focusing only on mental health disorders does not give the whole picture of the state of mental health among young people. A general problem is the predominant understanding of mental health as the absence of mental disorder. Risk factor research has focused on mental health problems rather than strengths and positive outcomes. Consideration of resilience has emerged from research indicating that a proportion of young people had a positive life trajectory despite having faced diverse potentially harmful life experiences. Worldwide up to $20 \%$ of children and adolescents suffer from disabling mental health problems: Mental health of adolescents and youth, it is a very problematic issue that is not expressed only with elements. This required a lot of analysis and statistic to arrive for a result which serves to us for knowing and preventive the problem. As an issue, we must identify two major part of this: What is mental health (definition, cause, diffusion, ages, and preventive)? And what can we do to improve the mental health of Adolescents and Youth (family, society, hospitals, school, and public places)? The estimates of psychological problems and disorders may therefore be higher than is reported in studies. On an individual level, mental health problems can have deteriorating effects on young people's social, intellectual, and emotional development and consequently on their future. At its worst, they can lead to loss of life. Suicide is one of the three leading causes of death in young people and a public health concern in many European countries. Besides the negative effects on an individual level, mental illness affects also many other spheres of life-family, friends, and society at large—causing costs not only in health care system. Furthermore, there are close links between child and adult mental illness—-the presence of mental illness during childhood may lead to up to 10 times higher costs during adulthood. Children are our future. Through well-conceived policy and planning, government can promote the mental health of children, for the benefit of the child, the family, the community, and society.
\end{abstract}

Keywords: mental health, adolescents, definition, mental illness, society, health care system, family

Valentina Telhaj, MSc, Psychology Consulting, Graduate School of Psychology Consulting, Departament of Psychology, European University of Tirana, Tirana, Albania. 


\section{Introduction}

Mental health can emerge in late childhood and early adolescence. Recent studies have identified mental health problems, in particular depression, as the largest cause of the burden of disease among young people.

Poor mental health can have import effect on the wider health and development of adolescents and is association with several health and social outcomes, such as higher alcohol, tobacco and illicit substances use, adolescent pregnancy, school dropout, and delinquent behaviors. There is growing consensus that healthy development during childhood and adolescence contributes to good mental health and can prevent mental health problems.

Enhancing social skills, problem-solving skills, and self-confidence can help prevent mental health problems, such as conduct disorders, anxiety, depression, and eating disorders as well as other risk behaviors including those that relate to sexual behavior, substance abuse, and violent behavior. Health workers need to have the competencies to relate to young people, to detect mental health problems early, and to provide treatments which include counseling, cognitive-behavioral therapy and where appropriate and psychotropic medication.

Child mental health policies are stimulated by the interaction of knowledge, public awareness, social mobilization, and advocacy. They are also influenced by other contextual issues, such as historic and existing health, social and educational policy, and services. The need to develop policy on child mental health has, sadly, been widely neglected, but is now recognized as a crucial first step in the development of accessible and effective services for children. As well as policy, detailed strategic action plans, identification of entry points and levers for change are all crucial.

\section{What Is Mental Health? Classic Version of the Hippocratic Oath}

(...) If I fulfill this oath and do not violate it, it may be granted to me to enjoy life and art, being honored with fame among all men for all time to come; if I transgress it and swear falsely, it may be the opposite of all this be my lot. (Edelstein, 1943) ${ }^{1}$

Mental health is more than just being free of a mental illness. It is more of an optimal level of thinking, feeling, and relating to others. What is mental illness? Mental illness refers to all of the diagnosable mental disorders. Mental disorders are characterized by abnormalities in thinking, feelings, or behaviors. Highly common, the individual can expect to meet the formal diagnostic criteria for some form of anxiety, depressive, behavioral, thought, or substance-abuse disorder during their lifetime. The common types of mental illness are anxiety, depressive, behavioral, and substance-abuse disorders, phobias, panic disorder, generalized anxiety disorder, and social anxiety disorder. Behavioral disorders are characterized by problems conforming to the tenets of acceptable behavior. Depressive disorders involve feelings of sadness that interfere with the individual's ability to function or, as with adjustment disorder, persist longer than most people experience in reaction to a particular life stressor.

\section{Definition}

Mental health has defined as "a state of successful performance of mental function, resulting in productive activities, fulfilling relationships with people, and the ability to adapt to change and to cope with adversity". ${ }^{2}$ It

\footnotetext{
1 The classical version of the Hippocratic Oath is from the translation from the Greek by Ludwig Edelstein.

${ }^{2}$ See http://www.medicinenet.com/script/main/art.asp?articlekey=20909.
} 
might seem easy to define mental health as the absence of mental illness; most experts agree that there is more to being mentally healthy. The state of being mentally healthy is enviable given the advantages it affords. For example, mentally healthy adults tend to report the fewest health-related limitations of their routine activities, the fewest full or partially missed days of work, and the healthiest social functioning (for example, low helplessness, clear life goals, high resilience, and high levels of intimacy in their lives). Mentally healthy individuals tend to have better medical health, productivity, and social relationships. Medications may play an important role in the treatment of a mental illness, particularly when the symptoms are severe or do not adequately respond to psychotherapy.

\section{Cause-What Are the Causes and Risk Factors for Mental Illness?}

Mental health disorders in children and adolescents are caused by biology, environment, or a combination of the two. Examples of biological factors are genetics and chemical imbalances in the body, and damage to the central nervous system, such as a head injury. Many environmental factors also can affect mental health, including exposure to violence, extreme stress, and the loss of an important person. There is no one test that definitively indicates whether someone has a mental illness. Therefore, health-care practitioners diagnose a mental disorder by gathering comprehensive medical, family, and mental-health information. Mental illness refers to all of the diagnosable mental disorders and is characterized by abnormalities in thinking, feelings, or behaviors. Some of the most common types of mental illness include anxiety, depressive, behavioral, and substance-abuse disorders. There is no single cause for mental illness. Rather, it is the result of a complex group of genetic, psychological, and environmental factors. While everyone experiences sadness, anxiety, irritability, and moodiness at times, moods, thoughts, behaviors, or use of substances that interfere with a person's ability to function well physically, socially, at work, school, or home are characteristics of mental illness. One frequently asked question about mental illness is if it is hereditary. Most mental disorders are not directly passed from one generation to another genetically, and there is no single cause for mental illness. Rather, it is the result of a complex group of genetic, psychological, and environmental factors. Genetically, it seems that more often than not, there seems to be a genetic predisposition to developing a mental illness. Everything from mood, behavioral and developmental, and thought disorders are thought to have a genetic risk for developing the condition.

\section{Ages}

The number of young people and their families who are affected by mental, emotional, and behavioral disorders is significant. It is estimated that as many as one in five children and adolescents may have a mental health disorder that can be identified and require treatment. Young people who experience excessive fear, worry, or uneasiness may have an anxiety disorder. "Anxiety disorders are among the most common of childhood disorders. According to one study of 9-year-old to 17 -year-old, as many as 13 of every 100 young people have an anxiety disorder", ${ }^{3} 100$ children may have major depression, and as many as eight of every 100 adolescents may be affected. ${ }^{4}$

\section{Preventive}

While medication can be quite helpful in alleviating and preventing overt symptoms for many psychiatric conditions, they do not address the many complex social and psychological issues that can play a major role in

\footnotetext{
${ }^{3}$ U.S. Department of Health and Human Services, 1999.

${ }^{4}$ National Institutes of Health, 1999.
} 
how the person with such a disease functions at work, at home, and in his or her relationships. The interventions are therefore seen by some as being forms of occupational therapy for people with mental illness. For example, treatment of bipolar disorder with medications tends to address two aspects: relieving already existing symptoms of mania or depression and preventing symptoms from returning.

\section{Hospitals}

Talk therapy (psychotherapy) is usually considered the first line of care in helping a person with a mental illness. It is an important part of helping individuals with a mental disorder achieves the highest level of functioning possible. Psychotherapies that have been found to be effective in treating many mental disorders include family focused therapy, psycho-education, cognitive therapy, interpersonal therapy, and social rhythm therapy.

\section{What Can We Do to Improve the Mental Health of Adolescents and Youth?}

\section{Family}

Families and communities, working together, can help children and adolescents with mental disorders. A broad range of services is often necessary to meet the needs of these young people and their families. Parents, practitioners, and policy-makers are recognizing the importance of young people's mental health. Youth with better mental health are physically healthier, demonstrate more socially positive behaviors and engage in fewer risky behaviors.

Conversely, youth with mental health problems, such as depression, are more likely to engage in health risk behaviors. Furthermore, youths' mental health problems pose a significant financial and social burden on families and society in terms of distress, cost of treatment, and disability. Most mental health problems diagnosed in adulthood begin in adolescence. Half of lifetime diagnosable mental health disorders start by age 14; this number increases to three fourths by age 24.6. (Knopf, Park, \& Mulye, 2008, p. 10)

The ability to manage mental health problems, including substance use issues and learning disorders, can affect adult functioning in areas, such as social relationships and participation in the workforce.

\section{Society}

Individuals with mental illness are at risk for a variety of challenges, but these risks can be greatly reduced with treatment, particularly when it is timely: Psycho-education, cognitive therapy, interpersonal therapy, and social rhythm therapy. Family focused therapy involves education of family members about the disorder and how to help (psycho-education), communication-enhancement training, and teaching family members problem-solving skills training. Psycho-education services involve teaching the person with the illness and their family members about the symptoms of the sufferer, as well as any warning signs (for example, change in sleep pattern or appetite, and increased irritability) that the person is beginning to experience another episode of the illness, when applicable. In cognitive behavioral therapy, the mental-health professional works to help the person with a psychiatric condition identify, challenge, and decrease negative thinking and otherwise dysfunctional belief systems. The goal of interpersonal therapy tends to be identifying and managing problems the sufferers of a mental illness may have in their relationships with others. Social rhythm therapy encourages stability of sleep-wake cycles, with the goal of preventing or alleviating the sleep disturbances that may be associated with a psychiatric disorder. 
The term "vulnerable child population" encompasses a broad spectrum of different individuals who are at greater risk of mental health problems for a non-exhaustive list of vulnerable populations. Those groups may have very different mental health needs, but often share experiences of stigma, discrimination, and/or difficulties accessing mental health services and promotion or preventive action. In addition, the available services may not be adapted to their specific needs. Some of these groups also constitute a low-prevalence group, and for this reason are further neglected. (Knopf, Park, \& Mulye, 2008, p. 22)

For example, in most of the surveys, there are problems with general indices representing the circumstances of children in minority groups—ethnic minorities, Roma, refugee/asylum seeker, and disabled children—which are too small in numbers to be represented in general samples of the population, and a tendency has also been noted for many of the indicators to relate only to the circumstances of older children. However, these low-prevalence groups usually have a greater need of attention compared to other more numerous groups which may already receive more attention. They are: children living in poverty homeless; children early school leavers; children experiencing bullying; children traveler; children juvenile offenders; children abandoned due to parental migration for employment, children physical, and learning disabled; children with mental disorders or drug abuse; children using alcohol; and abused children.

\section{School}

Children often feel sad, cry, or feel worthless then lose interest in play activities, or schoolwork declines. Physical well-being: Children may experience changes in appetite or sleeping patterns and may have vague physical complaints. They believe they are ugly, unable to do anything right, or that the world or life is hopeless. It also is important for parents and caregivers to be aware that some children and adolescents with depression may not value their lives, which can put them at risk for suicide.

The situation of young people is rapidly changing across the globe. The group of young people is less homogenous than the group of school aged children, and the life trajectory for young adults is not as

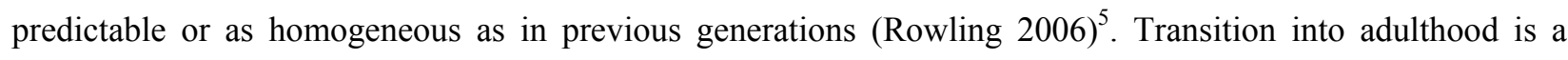
period which is determined by many changes. Adolescents and young adults are in a key phase of establishing independent identity, making educational and vocational decisions and lifestyle choices as well as forming interpersonal relationships. All of these have major long-term influences on the individual, particularly in terms of factors that influence mental health and well-being. Young people are particularly vulnerable to social exclusion, notably in the transition stage between education and employment. For example, leaving school early without access to full time work can lead to disconnection economically and socially and failure to develop a sense of the future. These young people form a specific category of "invisible" young people, as their possibilities and rights to a minimum income or health insurance are in many countries only minor (Stengård \& Appelqvist-Schmidlechner, 2010, p. 3). Schools play a major role in supporting young people with emotional and behavioural problems and are often where symptoms of mental disorders are first identified. A school staff member was among those to suggest that some help for emotional or behavioural problems was needed in two fifths $(40.5 \%)$ of cases. Just over one fifth (22.6\%) of young people who used health services had been referred by their school. Teachers and other school staff provided $18.9 \%$ of students with informal support for emotional and behavioural problems. This was higher (51.0\%) for students assessed as having a mental disorder. Of the four types of disorder, major depressive disorder had the greatest impact on school attendance. Students with

\footnotetext{
${ }^{5}$ Rowling, L. (2006). Adolescence and emerging adulthood (12-17 years and 18-24 years). In: Cattan, M. \& Tilford, S. (Eds.). Mental health promotion. A lifespan approach. The McGrawHill Education.
} 
this disorder averaged 20 days absent from school in the previous 12 months due to its symptoms. Major depressive disorder had the greatest impact on functioning at school, with one third (34.3\%) of students experiencing severe impact and another $34.1 \%$ a moderate impact due to this disorder. For adolescents, conduct disorders had almost the same level of impact ( $22.8 \%$ severe and $43.6 \%$ moderate), but conversely also had the highest proportion (21.8\%) for whom the disorder had no impact (Lawrence et al., 2015, p. 20).

\section{Public Places}

Stress has been found to be a significant contributor to the development of most mental illnesses, including bipolar disorder. For example, gay, lesbian, and bisexual people are thought to experience increased emotional struggles associated with the multiple social stressors associated with coping with reactions to their homosexuality or bisexuality in society. Unemployment significantly increases the odds ratio of an individual developing a psychiatric disorder. It almost quadruples the odds of developing drug dependence and triples the odds of having a phobia or a psychotic illness like schizophrenia. Being unemployed more than doubles the chances of experiencing depression, generalized anxiety disorder (GAD), and obsessive-compulsive disorder. Mental health problems of young people affect whole society, in spite of the fact that most children and adolescents perceive their health to be good; there is a sizeable minority of young people reporting their health to be either "fair" or "poor" and experiencing a number of recurring health complaints.

As mental health problems in adolescence tend to be under-recognized and undertreated (Sourander et al. 2004), estimates of psychological problems and disorders may therefore be higher than is reported in studies. On an individual level, mental health problems can have deteriorating effects on young people's social, intellectual and emotional development and consequently on their future. At its worst, they can lead to loss of life. Suicide is one of the three leading causes of death in young people and a public health concern in many European countries (WHO, 2001). Besides the negative effects on an individual level, mental illness affects also many other spheres of life-family, friends and society at large_causing costs not only in health care system. (Stengård \& Appelqvist-Schmidlechner, 2010, p. 7)

\section{Conclusion}

Young people can have mental, emotional, and behavioral problems that are real, painful, and costly. These problems, often called "disorders", are sources of stress for children and their families, schools, and communities. Monitoring systems are an important component of efforts to promote mental health, and prevent and treat mental health problems. Efforts promote a healthy adolescence and lay the groundwork for healthy adulthood.

The foundation for good mental health is laid in the early years and society as a whole benefits from investing in children and families. Fortunately, the majority of young people in the EU enjoy good mental health. However, on average, one in every five children and adolescents suffers from developmental, emotional, or behavioural problems and approximately $1 / 8$ have a clinically diagnosed mental disorder. Therefore, there is a clear and urgent need for development of effective policies and practices in enlarged Europe and for a creative process of interaction and a proactive exchange of information between European countries.

Naim Frasheri: "Give the child what he needs, and not what he wants"!

\section{References}

Adoleshentët dhe shëndeti mendor Udhëzime për familjet. (2006). Retrieved from https://zapdoc.tips/adoleshentt-dhe-shndeti-mendor.html

Edelstein, L. (1943). The Hippocratic Oath: Text, translation, and interpretation. Baltimore: Johns Hopkins Press. 
European Commission. (2009). Child and adolescent mental health in Europe: Infrastructures, policy and programmes. Retrieved from http://ec.europa.eu/health/ph_determinants/life_style/mental/docs/camhee_infrastructures.pdf

Knopf, D., Park, M. J., \& Mulye, T. P. (2008). The mental health of adolescents: A national profile. San Francisco: National Adolescent Health Information Center University of California.

Lawrence, D., Johnson, S., Hafekost, J., Boterhoven De Haan, K., Sawyer, M., ... Zubrick, S. (2015). The mental health of children and adolescents: Report on the second Australian child and adolescent survey of mental health and wellbeing. Australia: Commonwealth of Australia.

Stengård, E., \& Appelqvist-Schmidlechner, K. (2010). Mental health promotion in young people: An investment for the future. Retrieved from http://www.euro.who.int/_data/assets/pdf_file/0013/121135/E94270.pdf

World Health Organization (WHO). (2005). Child and adolescent mental health policies and plans. Geneva: World Health Organization (Mental Health Policy and Service Guidance Package). 